\title{
CONHECIMENTO SOBRE AS LEGISLAÇÕES E NORMAS REFERENTES ÀS BIFENILAS POLICLORADAS (PCB'S): STATUS ATUAL E APLICAÇÕES SOB A ÓTICA DA ENGENHARIA AMBIENTAL
}

\section{KNOWLEDGE ABOUT THE LAWS AND RULES RELATING TO THE POLYCHLORINATED BIPHENYLS (PCB'S ): CURRENT STATUS AND APPLICATIONS ON THE OPTICS OF THE ENVIRONMENTAL ENGINEERING}

\author{
Salatiel de Souza ${ }^{1}$; Welber Senteio Smith ${ }^{2}$ \\ ${ }^{1}$ Universidade de Sorocaba - UNISO - Sorocaba - Brasil \\ salaengambseg@bol.com.br \\ ${ }^{2}$ Universidade de Sorocaba - UNISO Programa de Mestrado em Processos Tecnológicos e \\ Ambientais \\ welber_smith@uol.com.br
}

\begin{abstract}
Resumo
A filosofia básica do gerenciamento dos resíduos de Ascaréis ( $P C B$ 's), que envolve o seu manuseio, estocagem, transporte e a destinação final, é eliminar e se não possível, reduzir ao máximo a exposição aos PCB's. A estratégia legal consiste em atuar diretamente na eliminação dos riscos que levem a exposição, atuando na prevenção através de processos adequados e dentro das Normas e Legislações, e nunca somente nas consequências.Neste sentido objetiva-se com este trabalho apresentar uma compilação do compendio legislativo/normativo, sua evolução histórica e o estado atual do cenário brasileiro frente a necessidade de inventariar o estoque de PCB's, contidos principalmente em equipamentos do setor elétrico como transformadores, disjuntores, capacitores, trocadores de calor, entre outros, o que é apenas um passo no gerenciamento desses resíduos, e realizar ao menos o compromisso do Brasil assumido na convenção de Estolcomo ( Estocolmo, 2001-2005).Aos envolvidos proprietarios dos passivos que herdaram de alguma forma, orientamos como proceder dentro do conhecimento das leis,porém o alto custo é fator impeditivo primordial do não cumprimento das leis e falta incentivo e colaboração da parte dos governos, que tem ciencia de tudo o que ocorre, mas faltam politicas favoraveis aos setores.
\end{abstract}

Palavras-chave: befenilas; legislação; equipamentos elétricos.

\section{Introdução}

O Brasil, país possuidor da mais elaborada e completa legislação referente ao Meio Ambiente em todas as suas formas de aplicação, é o signatário da Convenção de Estocolmo sobre Poluentes Orgânicos Persistentes, adotada, naquela cidade, em 22 de maio de 2001, que teve seu texto promulgado pelo decreto $\mathrm{n}^{0} 5.472$ em 20 de junho de 2005, tem o compromisso de fazer a gestão da destinação final de resíduos de Ascareis (PCB's) e eliminação desses resíduos, inclusive com data pré-estabelecida; 2025 (ESTOCOLMO, 2001-2005). 
Esta Convenção destaca-se por incluir no seu escopo a obrigação dos países Parte de adotarem medidas de controle relacionadas a todas as etapas do ciclo de vida - produção, importação, exportação, disposição e uso das substâncias classificadas como Poluentes Orgânicos Persistentes (POP's).

Os Poluentes Orgânicos Persistentes - POP's são substâncias químicas de alta persistência, capazes de serem transportados por longas distâncias, e de se acumularem em tecidos gordurosos dos organismos vivos, sendo tóxicos para o homem e para os animais. Os POP's circulam globalmente e podem causar danos nos diversos ambientes por onde passam.

Numa posição preventiva, a Convenção determina que os governos promovam as melhores tecnologias e práticas no seu campo tecnológico e previnam o desenvolvimento de novos POP's em suas plantas industriais. Indo mais além, define como seu objetivo final a eliminação total dos POP's, além de apresentar opções inovadoras e objetivas de ações em prol do desenvolvimento sustentável (VALENTE e TECORI, 2011).

A Convenção trata do gerenciamento, visando a eliminação, de substâncias químicas orgânicas que, segundo critérios definidos na própria convenção, são classificadas como sendo persistentes (não degradadas), passíveis de transporte a longa distância, pelo ar, água e solo, toxicologicamente preocupantes para a saúde humana e o meio ambiente.

Hoje estão identificadas 12 substâncias POP's, sendo oito agrotóxicos, dois produtos industriais, duas substâncias, dioxinas e furanos, que são formadas não intencionalmente em alguns processos industriais e, principalmente, durante a combustão de matéria orgânica na presença de cloro (ESTOCOLMO, 2001-2005).

\begin{tabular}{cc} 
Tabela 1- Substâncias poluentes orgânicos persistentes \\
\hline - Aldrin & - Hexaclorobenzeno (HCB) \\
- Clordano & - Mirex \\
- DDT & - Toxafeno \\
- Dieldrin & - Bifenilas Policloradas (PCB) \\
- Endrin & - Dioxina \\
- Furanos & - Heptacloro \\
\hline Fonte: Texto da Convenção de Estocolmo 2001
\end{tabular}

A Convenção de Estocolmo possui aspectos importantes no rol das convenções relacionadas à segurança porque prevê a eliminação e o banimento de substâncias químicas abrangidas pela Convenção, além da adoção de medidas de redução de eliminações não intencionais de dioxinas e furanos, produtos altamente tóxicos, de fontes prioritárias, como processos de queima de biomassa, de incineração de resíduos, de siderurgia e fundição, papel e celulose, indústrias químicas e outras.

A Convenção abrange também a identificação e eliminação de estoques de produtos agrotóxicos abrangidos pela mesma, bem como um inventário dos estoques de PCB's e equipamentos contaminados por este produto. 
Finalmente, a Convenção de POP's sela o compromisso das partes em identificar áreas contaminadas por POP's e promover sua reabilitação desde que entrou em vigor em 2004, após 50 países a ratificarem e atualmente, 164 Partes integram a Convenção de Estocolmo.

Os países deverão, ainda, elaborar Planos Nacionais de Implementação da Convenção (NIP), identificando prioridades, prazos e estratégias de cumprimento das obrigações constantes da Convenção (PNUD-GEF, 2009).

Constitui-se, portanto, num instrumento vinculante bastante complexo, que compreende substâncias altamente tóxicas e prejudiciais ao homem e ao meio ambiente, de grande interesse e acompanhamento por parte do setor industrial e da sociedade civil.

\section{Utilização dos PCB's e seus efeitos}

Em se tratando especificamente do elemento de estudo, as bifenilas policlorada (comumente chamadas de PCB's), principal constituinte do ascarel, óleo que foi muito utilizado em transformadores de energia elétrica, são compostos de origem antropogênica e foram sintetizados inicialmente na Alemanha, antes da virada do século 20 e produzidos em escala industrial a partir de 1929, pela Monsanto, nos Estados Unidos da América.

São compostos produzidos a partir da cloração catalítica de bifenilas, constituídos por dois anéis benzênicos unidos por uma ligação simples carbono-carbono, com até 10 átomos de cloro, os vários números e posições do cloro, em substituição ao hidrogênio nos anéis aromáticos da bifenila, resultam nas 209 possíveis estruturas químicas da bifenila, que denominados congêneres, estes compostos possuem baixo grau de reatividade, alta resistência elétrica, boas propriedades isolantes, não são inflamáveis e são estáveis ao calor e à pressão (VALENTE e TECORI,2011).

Devido a estas características foram amplamente aplicados como fluidos dielétricos e isolantes em transformadores e capacitores, dentre os possíveis congêneres de PCB's, 130 estão presentes nas misturas comerciais.

São compostos tóxicos que causam riscos à saúde, podem causar danos irreversíveis ao sistema nervoso central, considerados carcinogênicos afetam sobretudo, fígado, baço e rins, por serem bioacumulativos,pouco biodegradáveis e lipofílicos, acumulam-se em tecidos vegetais e animais.

No ecossistema terrestre, os organismos são contaminados nos mais baixos níveis tróficos, pela ingestão de solo e presas, além da absorção na pele ou inalação, as quais também podem ocorrer em algumas espécies, geralmente os organismos que se encontram no topo da cadeia alimentar sofrem mais riscos à exposição do PCB (PNUD-GEF, 2009) 


\section{Objetivo}

Como ainda não dispomos de estudos ou dados com relevância suficiente para se trabalhar esse problema, o presente trabalho se reporta totalmente as Legislações, Decretos, Normas e Instruções disponíveis, para mostrar uma visão crítica principalmente ao não cumprimento dessas leis, e o porquê isso se sucede no pais sob a óptica da Engenharia Ambiental.

Evidente que embora os documentos apresentados sejam oficiais, são restritos e suas amostras nesse trabalho não levarão á exposição dos seus detentores.

O fato de esse levantamento ser um trabalho real, e não apenas um auditoria para realização do trabalho de conclusão do curso de engenharia ambiental, nos levou a orientar os "donos" destes passivos a tomarem as medidas necessárias para manutenção e guarda dos equipamentos até sua destinação final para eliminação, conforme toda legislação aplicável.

\section{Materiais e métodos}

Foram utilizadas informações contidas em laudos de análises dos fluídos coletados e enviados ao laboratório certificado e autorizado conforme as Legislações pertinentes.

Além disso, foram consultadas Legislações, Normas, Decretos e Instruções referentes ás Bifelinas Policloradas (PCB's), e utilizadas como base legal dos levantamentos realizados nas auditorias em empresas de diversos seguimentos produtivos para validar esse trabalho diante do seu conteúdo, entre elas podemos citar:

a) Portaria Interministerial ( MIC/MI/MME ) No 19 de 29/01/81;

b) Instrução Normativa SEMA/STC/CRS/ No 001 de 10/06/83;

c) Lei federal 7347/85 (Lei de Ação Civil Pública);

d) Instrução Normativa SEMA STC/CRS-001, de 15/08/86;

e) Resolução CONAMA 06/88 (revogada pela Resolução CONAMA No 313 de 10/02);

f) Resolução CONAMA 02/91;

g) Decreto No 875 de 07/93;

h) Resolução CONAMA No 19 09/94;

i) Resolução CONAMA N ${ }^{\circ} 23$ de 12/96;

j) Medida Provisória MP 17 10/98;

k) Lei $n^{\circ} 9.605$ de 02/98;

1) Resolução CONAMA No 313, de 29/10/02 (Revoga Resolução CONAMA No 6 de 15/06/88);

m)Decreto Federal No 4.581 de 01/03;

n) Resolução CONAMA No 362 06/05; 
o) Decreto $N^{\circ}$ 5.472, 06/05 (Promulga o texto da Convenção de Estocolmo sobre Poluentes Orgânicos Persistentes, adotada, naquela cidade, em 22 de maio de 2001);

p) Lei Estadual (São Paulo) No 12288, de 22 de Fevereiro de 2006;

q) Lei No 12.305/2010 - Política Nacional de Resíduos Sólidos;

r) NBR 13741/96 - Destinação de bifenilas policloradas - procedimento;

s) NBR 10.004/04 Resíduos Sólidos;

t) NBR 8371/05 - Ascarel para transformadores e capacitores características e riscos;

u) NBR 13882/05 Líquidos isolantes elétricos determinação do teor de bifenilas policloradas;

v) Portaria $n^{\circ} 229$ de 24 de maio de 2011 atualiza NR-2.

Diante do exposto em toda a legislação e análises, coletamos informações em campo que nos dessem parametros do efetivo cumprimento normativo, a metodologia empregada foi a realização de auditorias nos sistemas elétricos de potência de algumas empresas possuidoras de subestações de energia elétrica, e consequentemente equipamentos isolados a óleo mineral, Figuras 1 e 2.

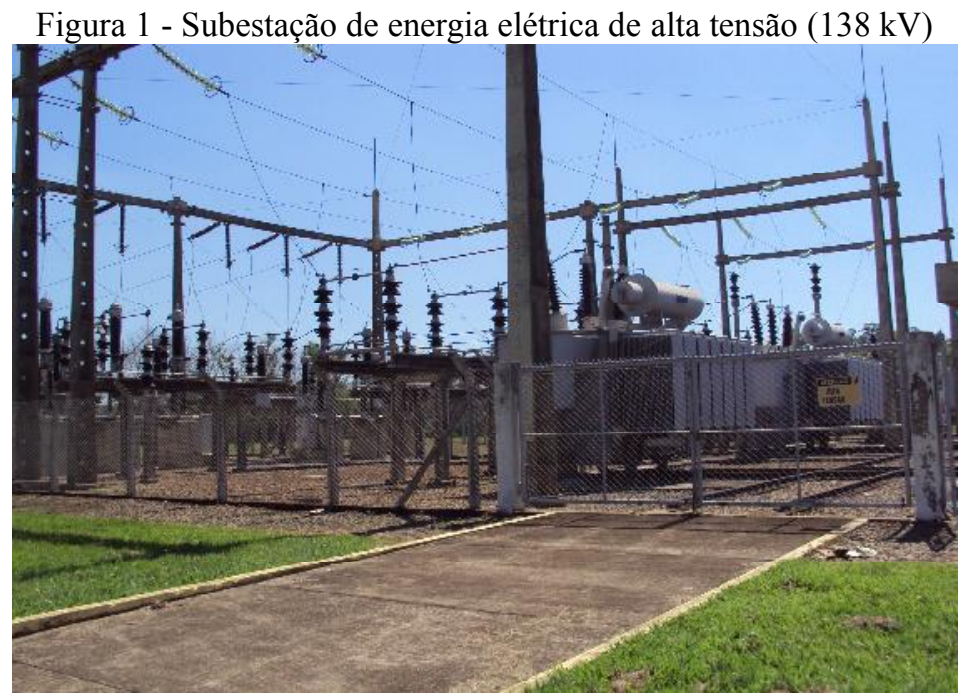

Figura 2 - Transformador de Potência Elétrica com identificação de substância perigosa (PCB)

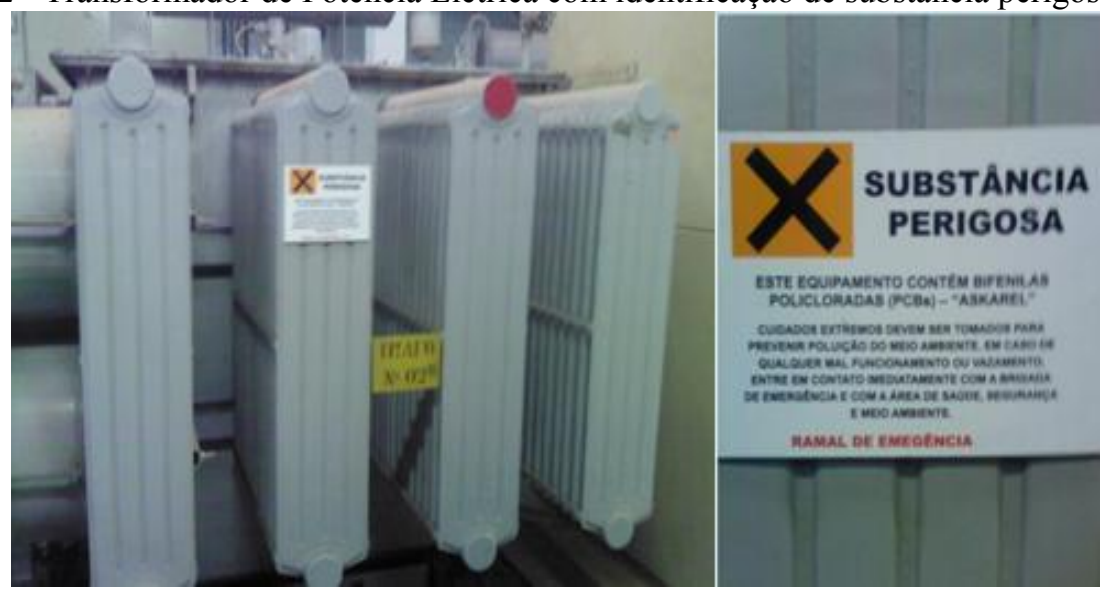

Fonte: Pesquisa de campo (2011)

Destes equipamentos; transformadores, disjuntores, capacitores, foram selecionados alguns 
transformadores e com uma equipe especializada coletamos amostras de óleo que foram enviadas a laboratórios credenciados para análises físico/química, cromatrografica e teor de PCB, Tabela 2 os resultados estão demonstrados neste trabalho, bem como registros fotográficos dos equipamentos, cópias dos protocolos de ensaios e fichas de análise com resultados.

Tabela 2 - Análise físico - química do fluído dielétrico ascarel

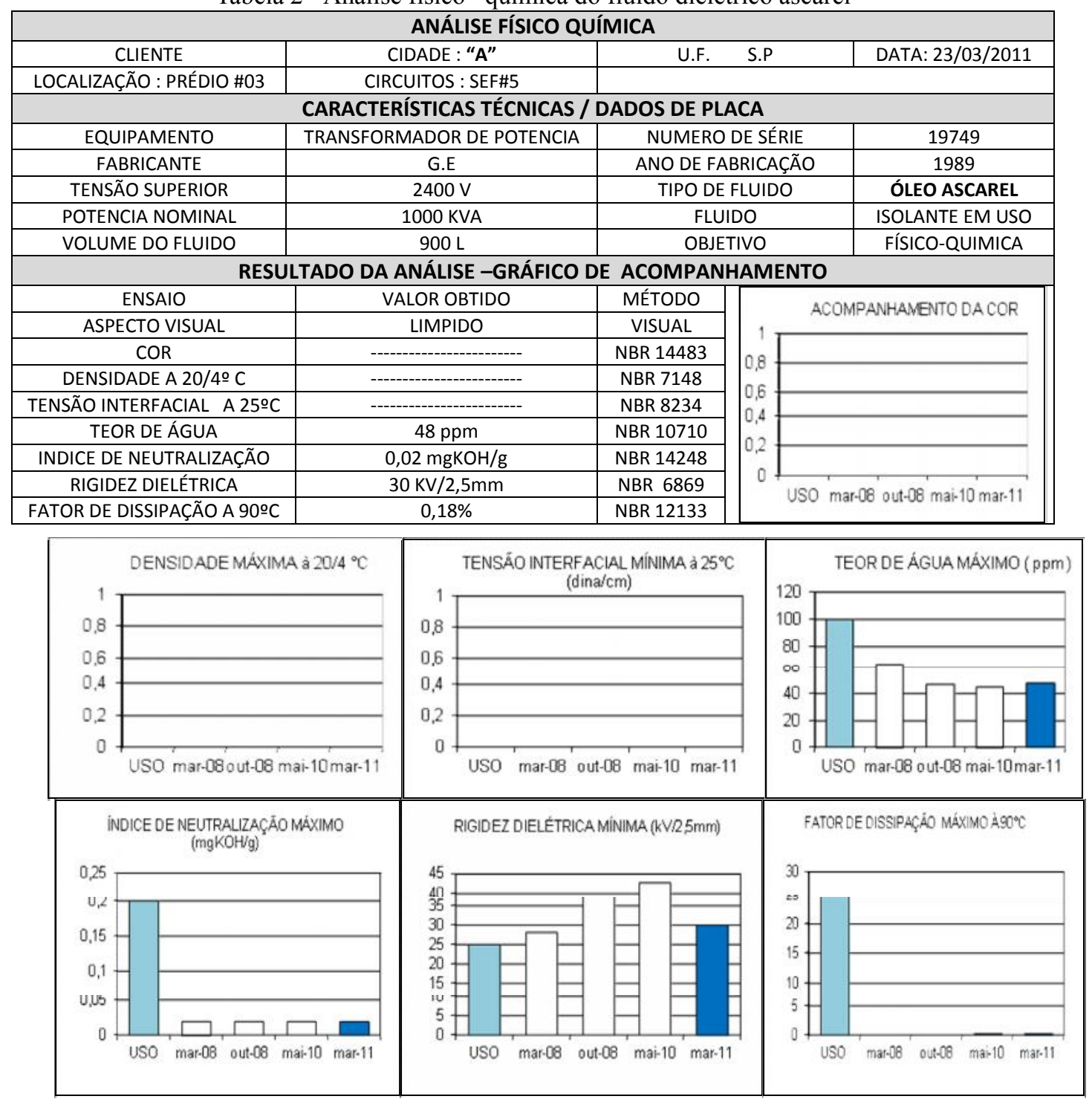

Fonte: Cotepe Engenharia Elétrica Ltda

Tendo como base o ano de 2011, para que as informações fossem as mais atuais possíveis e consideramos o prazo para cumprimento da lei estadual (São Paulo) $n^{\circ} 12.288$, de 22 de dezembro de 2006 que no cap. 11 Art 3 “ Dos Prazos”

A Destinação Final dos transformadores, capacitores e demais equipamentos elétricos contaminados com PCBs, que se encontram em operação e instalados em logradouros públicos, tais como, metrô, hospitais, salas de espetáculos, estádios de futebol, bancos, prédios públicos, etc., deve ser processada o mais breve possível, não devendo ultrapassar o mês de dezembro de 2010 .

A Tabela 3 abaixo resume os procedimentos de classificação dos equipamentos para o inventário nacional de bifenilas policloradas do Ministério do Meio Ambiente do Brasil, Secretaria 
de Mudanças Climáticas e Qualidade Ambiental, Departamento de Qualidade Ambiental na Indústria, o qual foi utilizado como base das auditorias, porém só foram verificados transformadores de energia elétrica (Guia para inventário de PCB's, PNUD, 2012).

Tabela 3 - Critérios definidos para levantamento dos dados em campo

\begin{tabular}{cc}
\hline Equipamento & Sem histórico de manutenção, pode ser considerado PCB, sem teste confirmatório. \\
\hline $\begin{array}{c}\text { Transformador } \\
\text { PCB, anterior a } \\
\text { 1989. }\end{array}$ & Com histórico de manutenção, verificar por teste rápido se o fluído dielétrico tem \\
concentração de PCB $>50 \mathrm{mg} / \mathrm{kg}$.
\end{tabular}

\section{Resultados e discussão}

Coletamos informações em grandes coorporações multinacionais da grande São Paulo e cidades do interior do estado com distancias de até $200 \mathrm{~km}$ da capital. Regiões tradicionalmente com forte tendencia industrial como as de Campinas, Sorocaba, Americana, Bauru, Riberão Preto estão entre os maiores dentetores desses resíduos,embora as demais regiões em que o agro-negócio é o tradicional também vivem o mesmo problema, as vezes até maiores pois são plantas antigas, algumas do início do séc XX, as tecnologias se atualizaram, mas os objetos de estudo desse trabalho são equipamentos com vida util longa e muitos ainda se encontram em operação.

Trataremos nesse trabalho as empresas pelas iniciais dos nomes ficticios (A,C,D) a quantidade levantada nos fornece um panorama expressivo do tamanho do problema, pois independente do segmento de atuação de qualquer empresa, se ela possui uma subestação de energia elétrica para alimentação do seu sistema, haverá no seu ambiente de manufatura ou atuação a distribuição dessa energia, que será feita por equipamentos como por exemplo os transformadores elétricos em quantidade, para elevação ou diminuição de potencia necessária para operação de Tornos ,Prensas, Fornos, Caldeiras, Moinhos, Injetoras, Chiller's (Ar Condicionado), Sistemas de Tecnologia, Extrusoras, Sopradoras, etc...

\subsection{Auditorias}

Auditoria realizada nos documentos de manutenção em subestações de energia elétrica da empresa "A", coleta de óleo, registros fotográficos, protocolos de ensaios e fichas de análise, com resultados laboratoriais, estão demonstradas como segue: 
Tabela 4 - Quantificação e identificação dos equipamentos e fluidos existente, empresa "A"

\begin{tabular}{|c|c|c|c|c|}
\hline Fabricante & Ano de Fabricação & Fluido Isolante & Volume L & $\begin{array}{c}\text { Potencia Nominal } \\
\text { kVA }\end{array}$ \\
\hline Induselet & 1979 & ascarel & 292 & 150 \\
\hline Induselet & 1979 & ascarel & 717 & 1000 \\
\hline Induselet & 1979 & ascarel & 717 & 1000 \\
\hline Induselet & 1979 & ascarel & 224 & 150 \\
\hline Induselet & 1979 & ascarel & 224 & 150 \\
\hline Induselet & 1979 & ascarel & 681 & 1000 \\
\hline Induselet & 1979 & ascarel & 681 & 1000 \\
\hline ITEL & 1975 & ascarel & 526 & 500 \\
\hline ITEL & 1975 & ascarel & 1084 & 750 \\
\hline Induselet & 1979 & ascarel & 217 & 150 \\
\hline Induselet & 1979 & ascarel & 514 & 500 \\
\hline Induselet & 1979 & ascarel & 514 & 500 \\
\hline Induselet & 1979 & ascarel & 765 & 1000 \\
\hline Induselet & 1979 & ascarel & 761 & 1000 \\
\hline G E & 1978 & ascarel & 990 & 1150 \\
\hline G E & 1978 & ascarel & 420 & 150 \\
\hline G E & 1978 & ascarel & 420 & 150 \\
\hline G E & 1978 & ascarel & 420 & 150 \\
\hline G E & 1978 & ascarel & 420 & 150 \\
\hline G E & 1978 & ascarel & 420 & 150 \\
\hline G E & 1981 & ascarel & 420 & 150 \\
\hline G E & 1981 & ascarel & 420 & 150 \\
\hline G E & 1981 & ascarel & 420 & 150 \\
\hline TOTAL & 23 unidades & ascarel & $12.267 \mathrm{~L}$ & \\
\hline
\end{tabular}

Fonte: Autoria própria (2011)

Tabela 5 - Identificação do transformador de alta tensão, com fluido isolante contaminado empresa "A"

\begin{tabular}{ccccc}
\hline Fabricante & Ano de Fabricação & Fluido Isolante & Volume L & Potencia Nominal kVA \\
\hline GE & 1972 & $\begin{array}{c}\text { Óleo mineral } \\
\text { Naftênico }\end{array}$ & 18.520 & 7.500 \\
\hline & & &
\end{tabular}

Fonte: Autoria própria (2011)

Um transformador de Alta tensão ( $138 \mathrm{kV}$ ) identificado na Tabela 5, apresentou em análise alto teor de bifenilas policloradas $(547 \mathrm{mg} / \mathrm{kg}$ ) portanto quase 11 vezes acima das $50 \mathrm{mg} / \mathrm{kg}$ determinados pela norma, ABNT/NBR 13882 esse equipamento passou por tratamento de termovácuo na tentativa de baixar esse percentual, mas a melhora foi insignificante chegou-se a 463 $\mathrm{mg} / \mathrm{kg}$. Conforme protocolo de resultado da Tabela 6, os gráficos em vermelho apresentam de forma clara o alto teor de PCB no fluido dielétrico isolante que nesse caso é óleo mineral naftênico.

Os ensaios são realizados conforme especificação da NBR 13882 método "A" (Potenciometria) e método "B" (Cromatografia Gasosa) conforme NBR 8371. Nota-se que os transformadores auditados não precisariam ter o fluido testado, pois os anos de fabricação já os condenariam, porém como temos histórico de manutenção, citamos nesse trabalho pelo fato de conhecermos e termos como comprovar por dados de placas dos transformadores e resultados das análises fisico/quimica que o fluido dielétrico é Ascarel. 
Tabela 6 - Protocolo do resultado da análise do teor de bifenilas policloradas

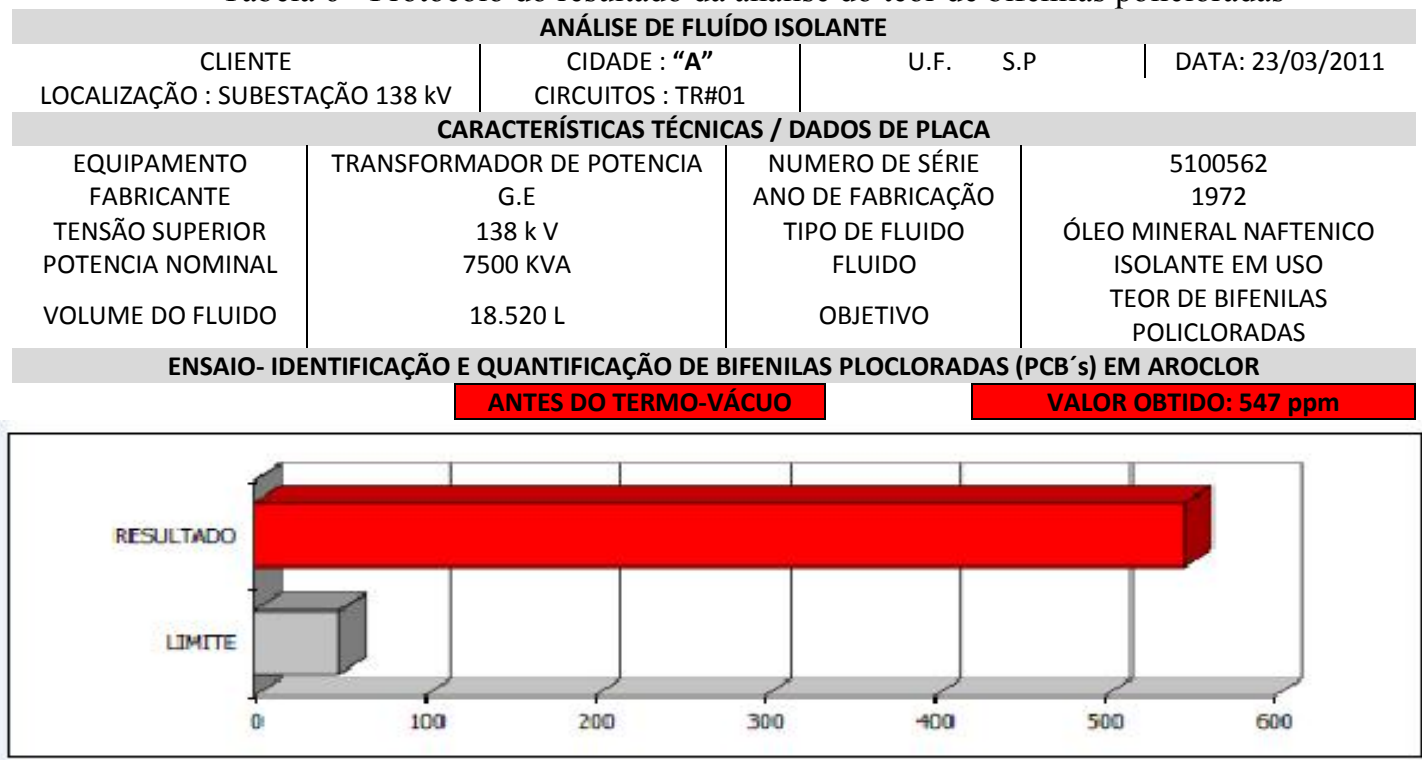

ENSAIO- IDENTIFICAÇÃO E QUANTIFICAÇÃO DE BIFENILAS PLOCLORADAS (PCB's) EM AROCLOR

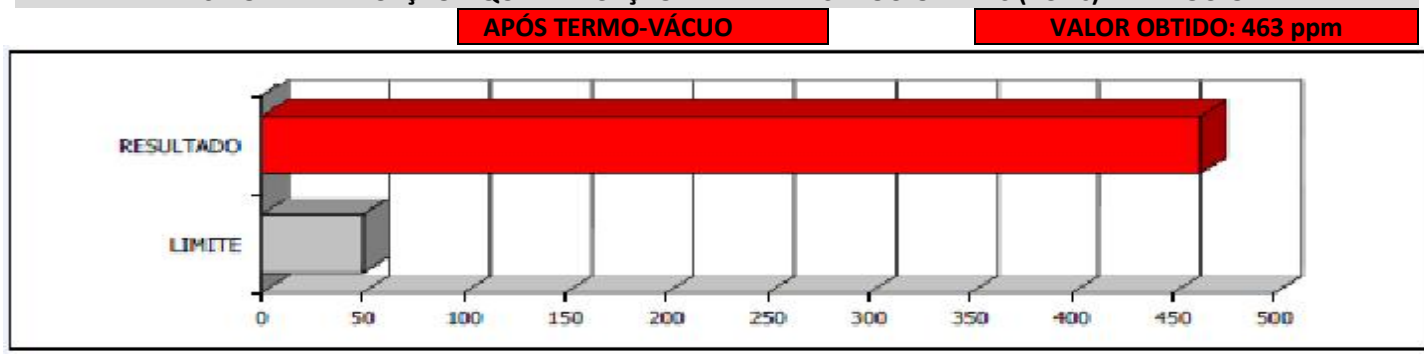

Fonte: Cotepe Engenharia Elétrica Ltda

Vale frisar que transformadores de Alta Tensão Figura 3, não utilizavam ascarel como fluido isolante, esse equipamento teve o óleo mineral naftenico contaminado, bastante provável que seja por bombas de termo-vácuo que foram utilizadas na tentativa de tratar ascarel e posteriormente as usaram para tratar óleo mineral naftenico sem a assepsia necessária, o que causou a contaminação e isso ocorreu em muitas ocasiões o que justifica o fato de encontrarmos tantos equipamentos com altos teores de PCB's.

Figura 3 - Transformador de potência elétrica de alta tensão (A.T) $138 \mathrm{kV}$ com óleo mineral contaminado

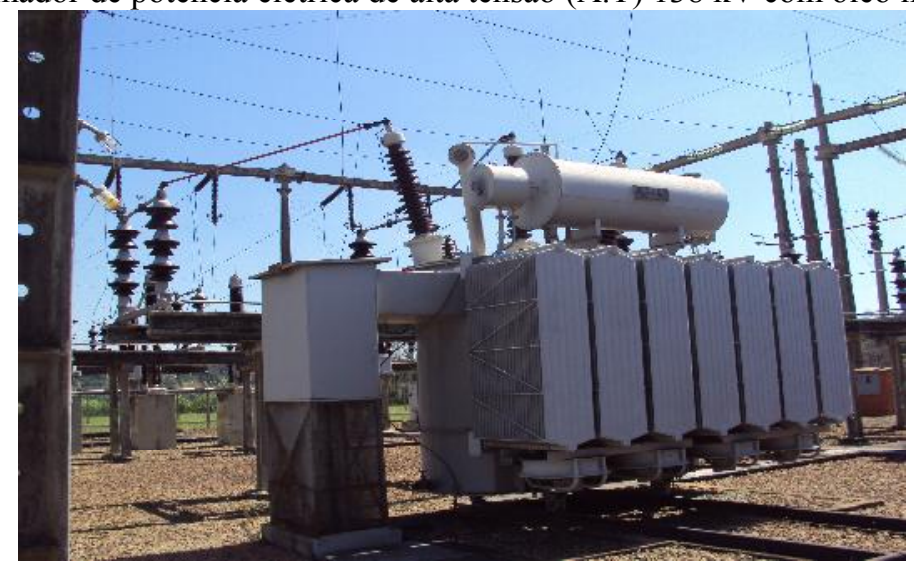

Fonte: Pesquisa de campo (2011) 
Auditoria realizada nos documentos de manutenção em subestações de energia elétrica da empresa "C" Tabela 7 , coleta de óleo, registro fotográficos, protocolos de ensaios e fichas de análise, com resultados laboratoriais estão demonstrados como segue:

Tabela 7 - Quantificação e identificação dos equipamentos e fluidos existentes, empresa "C"

\begin{tabular}{ccccc}
\hline Fabricante & Ano de Fabricação & Fluido Isolante & Volume L & Potencia Nominal kVA \\
\hline Induselet & 1981 & ascarel & 1300 & 2000 \\
Induselet & 1981 & ascarel & 1300 & 2000 \\
Induselet & 1981 & ascarel & 1300 & 2000 \\
TOTAL & $\mathbf{0 3}$ unidades & ascarel & $\mathbf{3 9 0 0 ~ L}$ & \\
\hline
\end{tabular}

Fonte: Autoria própria (2011)

Nessa amostra chegamos ao total de 3900 litros de ascarel, ou seja, próximo de 4 t, em equipamentos que estão em operação.

Abaixo, protocolo de ensaio dos óleos coletados que foi enviado ao laboratório credenciado e que mantém parceria com a empresa de manutenção para realização desses ensaios que chamamos de “ Análise de Fluido Isolante", nas Tabelas 8 e 9 encontram-se identificação dos transformadores e fluidos verificados, quantificados e registro do agravante dessa mesma amostra que embora seja menor que a da empresa "A" encontramos equipamentos com vazamento de Ascarel Figuras 4 (a e b).

Tabela 8 - Protocolo de ensaio do transformador (medições/testes elétricos) e dados de placa

\begin{tabular}{|c|c|c|c|c|}
\hline \multicolumn{5}{|c|}{ Análise de fluído isolante } \\
\hline Cliente & Cidade : "c" & u.f. & s.p & $/ 03 / 2011$ \\
\hline Localização : sube & Circuitos : $\operatorname{tr} \# 02$ & & & \\
\hline \multicolumn{5}{|c|}{ características técnicas / dados de placa } \\
\hline Equipamento & Transformador de potência & \multicolumn{2}{|c|}{ Número de série } & 21153 \\
\hline Fabricante & Induselet & \multicolumn{2}{|c|}{ Ano de fabricação } & 1981 \\
\hline Tensão superior & $13860 / 13530 / 12870 / 12540 \mathrm{v}$ & \multicolumn{2}{|c|}{ Tipo de fluido } & Ascarel \\
\hline Tensão inferior & $2400 / 1385 \mathrm{v}$ & \multicolumn{2}{|c|}{ Impedancia } & $5,61 \%$ \\
\hline Potência nominal & $2000 \mathrm{Kva}$ & \multicolumn{2}{|c|}{ Frequência } & $60 \mathrm{hz}$ \\
\hline Volume do fluido & 13001 & & & \\
\hline
\end{tabular}

Fonte: Autoria própria (2011)

Tabela 9 - Protocolo de inspeção visual do transformador

\begin{tabular}{|c|c|c|c|c|c|c|c|}
\hline \multicolumn{8}{|c|}{$\begin{array}{c}\text { TRANSFORMADOR DE POTENCTA } \\
\text { MEDIA TENSATO }\end{array}$} \\
\hline \multicolumn{3}{|l|}{ CLIENTE: } & \multirow{2}{*}{\multicolumn{5}{|c|}{$\begin{array}{l}\text { CIDADE: } \\
\text { CIRCUITO: UCP's Transformador TR - } 02 \pi \text { TA } 7 \text {-9150 }\end{array}$}} \\
\hline \multicolumn{3}{|l|}{ LOCALIZAÇĀO: } & & & & & \\
\hline \multicolumn{8}{|c|}{ IUSPECÁO DAS CONDICÖES GERAIS DO EQUIPAMENTO } \\
\hline \multirow{8}{*}{$\begin{array}{l}\text { - VERIFICADO } \\
\text { - AUSENTE } \\
\text { - OBSERVAÇAOO }\end{array}$} & $\checkmark$ & - & TANQUE PRINCIPAL & $\checkmark$ & \multirow{8}{*}{$\begin{array}{l}- \\
- \\
- \\
- \\
- \\
- \\
- \\
-\end{array}$} & COMU & T. DE TAPES \\
\hline & $\checkmark$ & -1 & RADIADORES & $\checkmark$ & & TERM & METRO DE ÓLEO \\
\hline & 0 & - & VÁLVULAS E REGISTROS & $\checkmark$ & & \multirow{6}{*}{\multicolumn{2}{|c|}{$\begin{array}{l}\text { INDICADOR NIVEL DE ÓLEO } \\
\text { VÁLVULA DE SEGURANCAA } \\
\text { INDICADOR DE PRESSÃO } \\
\text { RELE DE GÁS } \\
\text { LIMPEZA DOS CONTATOS } \\
\text { OUTROS } \\
\end{array}$}} \\
\hline & $\checkmark$ & & BUCHAS ISOLANTES PRIM. & $\checkmark$ & & & \\
\hline & $\checkmark$ & & BUCHAS ISOLANTES SEC. & - & & & \\
\hline & $\checkmark$ & & ATERRAMENTOS & - & & & \\
\hline & $\checkmark$ & & REAPERTO & - & & & \\
\hline & - & & REPOSICC̃̃O & - & & & \\
\hline \multicolumn{8}{|l|}{ OBSERVACŌES GERAIS } \\
\hline \multirow{2}{*}{\multicolumn{8}{|c|}{ - A placa de identificação encontra-se solta. }} \\
\hline & & & & & & & \\
\hline \multicolumn{8}{|c|}{ - As proteçóes intrisecas encontram-se desativadas. } \\
\hline \multicolumn{8}{|c|}{ - Sinais de vazamento de fluido isolante pelos parafusos da tampa superior. } \\
\hline \multicolumn{8}{|c|}{ - Sinais de vazamento de fluido isolante pelo registro inferior. } \\
\hline
\end{tabular}

Fonte: Cotepe Engenharia Elétrica Ltda 
Figura 4 (a e b) - Sinais de vazamento do fluido isolante pelos parafusos da tampa superior e pelo registro inferior
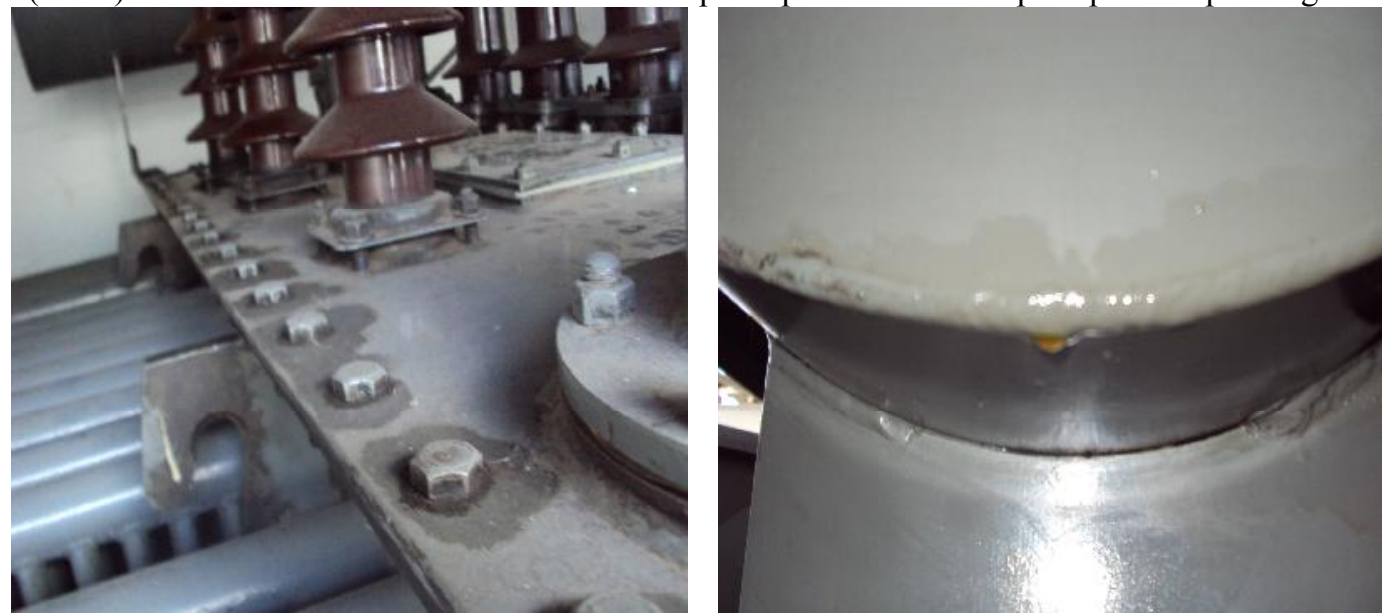

Fonte: Pesquisa de campo (2011)

Auditoria realizada nos documentos de manutenção em subestações de energia elétrica da empresa "D" Tabela 10, coleta de óleo Figura 6, protocolos de ensaios com resultados laboratoriais, estão demonstradas como segue:

Tabela 10 - Quantificação e identificação do equipamento e fluidos existentes, empresa "D"

\begin{tabular}{ccccc}
\hline Fabricante & Ano de Fabricação & Fluido Isolante & Volume L & Potencia Nominal kVA \\
\hline União & 1983 & Óleo mineral & 10040 & 10000 \\
\hline
\end{tabular}

Fonte: Autoria própria (2011)

Observa-se muito claramente pela identificação do transformador que, embora seja de outro fabricante e 11 anos mais novo que o equipamento da empresa " $A$ " esse da empresa " $D$ " possui as mesmas características e o volume de fluido para uma única amostra bastante grande, e da mesma forma foi também contaminado, o protocolo de resultado da análise de teor de PCB's Tabela 11, mostra o percentual de $56,7 \%$ quando o máximo conforme NBR 8371 deveria ser $50 \%$.

Tabela 11 - Protocolo de resultado da análise de teor de PCB's

\begin{tabular}{|c|c|c|c|}
\hline \multicolumn{4}{|c|}{ DEPARTAMENTO TÉCNICO LABORATÓRIO } \\
\hline \multicolumn{4}{|c|}{ 04/10/2011- Relatório de análise de teor de PCB's em óleo isolante } \\
\hline \multicolumn{4}{|l|}{ Cliente: Cotepe Engenharia Elétrica ltda } \\
\hline \multicolumn{2}{|l|}{ Equipamento: TRANSFORMADOR (T) } & \multicolumn{2}{|c|}{ Número de série: 501087} \\
\hline \multicolumn{2}{|l|}{ Fabricante: União } & \multicolumn{2}{|c|}{ Identificação: } \\
\hline \multicolumn{2}{|l|}{ Tensão primária: $88 \mathrm{Kv}$} & \multicolumn{2}{|c|}{ Ano de fabricação: 1983} \\
\hline \multicolumn{2}{|l|}{ Tensão secundária: Kv } & \multicolumn{2}{|c|}{ Local: associação } \\
\hline \multicolumn{2}{|l|}{ Potência: $10000 \mathrm{Kva}$} & \multicolumn{2}{|c|}{ Sistema de preservação: não informado } \\
\hline \multicolumn{2}{|l|}{ Volume: 10040 litros } & \multicolumn{2}{|c|}{ CDC - óleo: não informado } \\
\hline \multicolumn{4}{|l|}{ Sistema de refrigeração: não informado } \\
\hline \multicolumn{4}{|l|}{ Número da coleta: 111798} \\
\hline \multicolumn{4}{|l|}{ Data da coleta: $08 / 09 / 2011$} \\
\hline \multicolumn{4}{|l|}{ Temp termômetro do óleo (.C): 27} \\
\hline \multicolumn{4}{|l|}{ Umidade relativa do ar (\%): 34} \\
\hline \multicolumn{4}{|l|}{ Ponto de amostragem: Reg. inferior } \\
\hline Unidade & Método & 151903 & \\
\hline Aspecto visual & Visual & Preventiva & NBR-10576 (30/11/06) \\
\hline Teor de pcb & Nbr 13882 & Límpido & Límpido \\
\hline & & 56.7 & 50 máximo (NBR 8371) \\
\hline
\end{tabular}




\section{DIAGNÓSTICO}

Ensaio realizado conforme nbr-13882:2008 "líquidos isolantes elétricos - determinação do teor de bifenilas policloradas (PCB) - cromatografia gasosa". O limite de quantificação do método é de $2.0 \mathrm{mg} / \mathrm{Kg}$. Líquido isolante classificado como contaminado com PCB segundo nbr-8371:2005. Nessas condições devem-se observar os procedimentos especiais para fins de manuseio, acondicionamento, transporte e manutenção nela descritos.

Resultado expresso em aroclor 1242/1254/1260.

Resultados obtidos referem-se estritamente à amostra recebida em nosso laboratório.

Fonte: Cotepe Engenharia Elétrica Ltda

Figura 6 - Realização de coleta de óleo dos transformadores para análise laboratorial
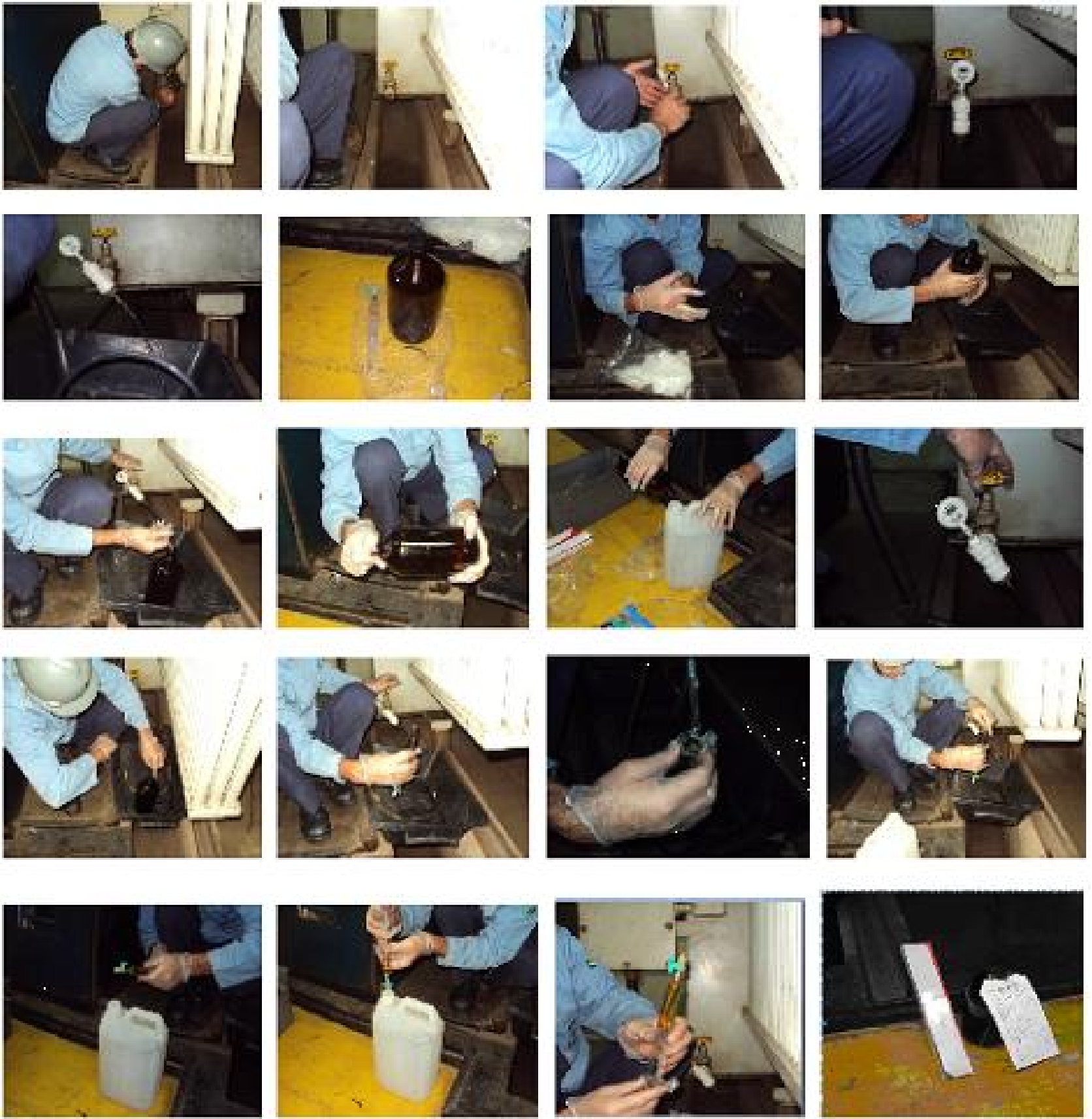

Fonte: Autoria própria (2011) 


\section{Conclusões}

\begin{tabular}{|c|c|c|c|c|}
\hline & Amostras & Equipamentos & Fluido Isolante & Total L \\
\hline & $\begin{array}{c}1 \text { a Amostra } \\
\text { Empresa "A" }\end{array}$ & 23 unidades & ascarel & $12.267 \mathrm{~L}$ \\
\hline & $\begin{array}{c}2^{\mathrm{a}} \text { Amostra } \\
\text { Empresa "A" }\end{array}$ & 01 unidade & (PCB) & $18.520 \mathrm{~L}$ \\
\hline & $\begin{array}{c}3^{\mathrm{a}} \text { Amostra } \\
\text { Empresa "C" }\end{array}$ & 03 unidades & ascarel & $3.900 \mathrm{~L}$ \\
\hline & $\begin{array}{c}4^{\mathrm{a}} \text { Amostra } \\
\text { Empresa "D" }\end{array}$ & 01 unidade & PCB's & $10.040 \mathrm{~L}$ \\
\hline TOTAL & Amostras & 28 unidades & Ascarel+PCB's & $44.727 \mathrm{~L}$ \\
\hline
\end{tabular}

O resultado das amostras nos apresenta um total de 44.727 L (Volume) de PCB's, ou seja aproximadamente $45 \mathrm{t}(\mathrm{Kg})$.

A pesquisa resulta de uma amostragem pequena mas em volume de PCB's altissima pelos problemas que podem causar a saúde e ao meio ambiente, essas empresas como já citadas são grandes consumidoras de energia elétrica, em sua maioria sites antigos, porém em atividade produtiva, o que leva os detentores desses problemas a praticamente ficarem impossibilitados de investir na resolução dessas causas uma vez que os custos são muito altos.

Acreditamos ter demonstrado com esse trabalho que o problema é grave e infelizmente não é tratado com deveria conforme toda legislação estabelecida e o compromisso firmado pelo nosso pais de erradicar esse problema da nação e por consequência levar aos demais países a maneira que se deve proceder a respeito dos PCB's.

A citação se deve especialmente por que está demonstrado que existe o problema com os PCB's, existe legislação federal, estadual e em alguns casos municipal, decretos, instruções normativas, procedimentos, a participação de órgãos internacionais como o "Programa das Nações Unidas para o Desenvolvimento"(PNUD), porém nas auditorias, levantamentos e contatos realizados com os órgãos oficiais não encontrei evidencia de prontuários elaborados ou apresentados ás autoridades e não existe nenhum orgão que hoje fiscalize as empresas.

As respostas que obtivemos quando procuramos pelas autoridades publicas, as quais não citaremos aqui por não poder evidenciá-las e por ética, foram sempre as mesmas; não possuem efetivos e nem conhecimento técnico que os levem a fiscalizar de maneira correta essas questões.

O próprio autor da lei estadual de São Paulo, 12.288 de 22 de fevereiro de 2006, que:

Dispõe sobre a eliminação controlada dos PCBs e dos seus resíduos, a descontaminação e da eliminação de transformadores, capacitores e demais equipamento elétricos que contenham PCBs, e dá providências correlatas.

fez um requerimento a camara municipal de São Paulo solicitando a apresentação de documentos das empresas que ora deveriam ter sido vistoriadas e entregues seus inventários ou sido multadas se 
não o fizessem, como escopo da própria lei, após 05 anos do referido requerimento, ainda não obteve resposta.

Isso demonstra que não se tem feito cumprir as leis e não existe a preocupação necessária a respeito de tão grave questão ambiental de ninguem, nem dos proprietarios dos equipamentos e residuos,pois tem certeza da impunidade, nem das autoridades por não possuirem ferramentas para realização do cumprimento dessas leis.

\title{
Agradecimento
}

O autor agradece a Cotepe Engenharia Elétrica Ltda (Sorocaba - SP) pela colaboração e disponibilidade de acessos aos clientes e colaboradores nessas auditorias.

\begin{abstract}
The basic philosophy of the management of waste from Ascareis (PCB's ), involves your handling, storage, transportation and final disposal, is delete and if not possible, to reduce the maximum exposure to PCB's .The legal strategy consists in directly act on elimination of the risks that lead to exposure, acting on prevention through appropriate processes and within the Rules and Laws, and never only in consequences.In this sense it is aimed with this work present a compilation of compendium legislative/normative, its historical development and the current state of the Brazilian scenario facing the need to inventory the inventory of PCB's, contained mainly in equipment of electric sector such as transformers, circuit breakers, capacitors, heat exchangers, among others, which is only a step on managing these wastes,and perform at least the commitment of Brazil assumed in the convention of Estolcomo ( Stockholm, 2001-2005). Those involved owners of liabilities that inherited in some way, we told how to proceed within the knowledge of the laws, but the high cost is the primary factor that impeded non compliance with laws and lack encouragement and collaboration on the part of governments that have science of everything that happens, but lack of policies favorable to sectors.
\end{abstract}

Key-words: befenilas; legislation; electrical equipment.

\section{Referências}

ASSOCIAÇÃO BRASILEIRA DE NORMAS TÉCNICAS. NBR 8371: Ascarel para transformadores e capacitorescaracterísticas e riscos. Brasil, 2005.

ASSOCIAÇÃO BRASILEIRA DE NORMAS TÉCNICAS. NBR 10576: Óleo mineral isolante de equipamentos elétricos- Diretrizes para supervisão e Manutenção. Brasil, 2006.

ABRAMOWICZ, D. A., aerobic and anaerobic PCB biodegradation in the environment; in the conference on biodegradation; its role in reducing toxicity and exposure to environmental contaminants helds, 26-28 abril 1993 in Research Triangle Park, North Carolina.

ASSOCIAÇÃO BRASILEIRA DE NORMAS TÉCNICAS. NBR 13882: Determinação do teor de bifenilas policloradas (PCB). Brasil, 2008.

BORJA, J.; TALEON, D. M., AURESENIA, J., GALLARDO, S. Polichlotinated biphenyls and their biodegradation . Process biochemistry, v. 40, p. 1999-2013, 2005.

BRASIL, MINISTÉRIO DO MEIO AMBIENTE, Secretaria de Mudanças Climáticas e Qualidade Ambiental, Departamento de Qualidade Ambiental na Indústria: Guia para o inventário nacional de bifenilas policloradas (PCB) no setor elétrico 1a ed. - Brasília: 2012. 
CORRÊA, R. C. Degradação de bifelina policlorada e caracterização da comunidade microbiana de reator anaeróbio com biofilme. USP, São Carlos, 2011.

DESTOXIFICAÇÃO DE BIFENILAS POLICLORADAS ATRAVÉS DE HIDRODESCLORAÇÃO CATALÍTICA In: Anais da 30a. Reunião Anual da SBQ, 2007.

DECRETO n 5.472, de 20 de junho de 2005 (Promulga o texto da Convenção de Estocolmo sobre Poluentes Orgânicos Persistentes).

DECRETO nº 875 de 07/93 (Promulga o texto da Convenção sobre o Controle de Movimentos Transfronteiriços de Resíduos Perigosos e seu Depósito).

ERICKSON, M. D. Analitical Chemistry of PCBs. Lewis Publishers, Chelsea, 1986.

HUTZINGer, O.; SAFE S.; ZITKO, V. The Chemistry of PCBs. CRC Press, New York, 1974, p 8.

INSTRUÇÃO NORMATIVA SEMA/STC/CRS/ nº 001 de 10/06/83.

LEI FEDERAL n $^{\mathbf{7}} \mathbf{7 3 4 7 / 8 5}$ (Lei de Ação Civil Pública).

LEI FEDERAL $\mathbf{n}^{\circ}$ 9.605 de 02/98 (Dispõe sobre as sanções penais e administrativas derivadas de condutas e atividades lesivas ao meio ambiente, e dá outras providências).

LEI ESTADUAL (São Paulo) N 12288, de 22 de Fevereiro de 2006 (Dispõe sobre a eliminação controlada dos PCBs e dos seus resíduos, a descontaminação e da eliminação de transformadores, capacitores e demais equipamento elétricos que contenham PCBs, e dá providências correlatas).

LEI FEDERAL n⿳ 12.305/2010 - Política Nacional de Resíduos Sólidos (Institui a Política Nacional de Resíduos Sólidos; altera a Lei n⿳0 9.605, de 12 de fevereiro de 1998; e dá outras providências).

NBR n 10.004/04 Resíduos Sólidos (dispõe sobre a classificação dos resíduos sólidos quanto aos seus riscos potenciais ao meio ambiente e à saúde pública).

NBR n 13882/05 -Líquidos isolantes elétricos - determinação do teor de bifenilas policloradas (PCB).

NBR $\mathbf{n}^{\mathbf{0}}$ 8371/05 - Ascarel para transformadores e capacitores características e riscos.

POLICARPO, N.A. Tratamento de solos contaminados com bifelinas policloradas (PCB's) USP, São Paulo, 2008.

PENTEADO, J. C. P.; VAZ, J. M. O legado das bifelinas policloradas (PCBs). Quimica Nova, v. 24, nº 3, 390-398, 2001. cross ref

PNUD - PROGRAMA DAS NAÇÕES UNIDAS PARA O DESENVOLVIMENTO Global Environment Facility São Paulo, 2009-2011.

PORTARIA INTERMINISTERIAL (MIC/MI/MME) n⿳0 19 de 29/01/81.

PORTARIA N 229 de 24 de maio de 2011 atualiza (Norma Regulamentadora) NR -26.

RESOLUÇÃO CONAMA No 313, de 29/10/02 Dispõe sobre o Inventário Nacional de Resíduos Sólidos Industriais (REVOGA RESOLUÇÃO CONAMA No 6 de 15/06/88).

RESOLUÇÃO CONAMA No 19 09/94 (Art. 1º Fica autorizada, em caráter de excepcionalidade, a exportação de resíduos perigosos contendo bifenilas policloradas - PCBs, sob todas as formas em que se apresentem).

RESOLUÇÃO CONAMA N²3, de 12/12/1996 (Dispõe sobre as definições e o tratamento a ser dado aos resíduos perigosos, conforme as normas adotadas pela Convenção da Basiléia sobre o Controle de Movimentos Transfronteiriços de Resíduos Perigosos e seu Depósito).

RESOLUÇÃO CONAMA Nº 362 06/05 (Dispõe sobre o recolhimento, coleta e destinação final de óleo lubrificante usado ou contaminado).

TIEDJE, J. M.; QUENSEN III , J. F.; CHEE STANFORD, J.; SCHIMEL, J. P.; BOYD, S. A. Microbial reductive 
dechlorination of PCBs. Biodegradation, v. 3, p. 231-240, 1993.

VALE, L. A. S.; MORAES, J. E. F.; BAZITO, R. C. Destoxificação de Bifenilas Policloradas através de Hidrodescloração Catalítica. In: anais da $30^{\text {a }}$. Reunião

VALENTE, R. P. CASTANHO, Destinação Final Resíduos de Ascaréis - PCB’s São Paulo, 2011. Apostila complementar da palestra "Alternativas de Destinação Final de Resíduos com ascaréis (PCBs)".

\section{Dados dos autores}

Nome Completo: Salatiel de Souza

Filiação Institucional: Universidade de Sorocaba - UNISO

Departamento: Graduação

Função ou cargo ocupado: Estudante

Endereço completo para correspondência: Rua Romeu de Melo 137- Jardim Marco Antonio Sorocaba - SP - Brasil CEP 18080-290 Telefones para contato (15) 32341537 (15) 981280700 e-mails: salaengambseg@bol.com.br/salatiel@cotepe.com.br

\section{Nome Completo: Welber Senteio Smith}

Filiação Institucional: Universidade de Sorocaba - UNISO

Departamento: Engenharia Ambiental/Programa de Mestrado em Processos Tecnológicos e Ambientais

Função ou cargo ocupado: Titular

Endereço completo para correspondência: Sorocaba - SP - Brasil

e-mail: welber_smith@uol.com.br

Submetido em: 15/04/2014

Aceito em: 17/12/2014 